\title{
Antropologilandia o una personal visión de la disciplina ${ }^{1}$
}

\author{
FERNANDO MONGE \\ Instituto de Historia, CSIC
}

Hace no muchos años un conocido me preguntó cuál era mi actividad profesional, a lo que contesté que antropólogo; encantada, esa persona me explicó su entusiasmo ante mi disciplina por su gran amor e interés hacia los animales. Tengo la sensación que en ese momento no nos estábamos refiriendo al mismo tipo de animales.

Para mí, como para muchos de mis compañeros, no creo que esta anécdota sea una ocurrencia insólita en sus vidas, más bien, un claro reflejo del relativo desconocimiento que existe sobre la antropología en nuestro país. Siendo un poco más crítico podría añadir que ese desconocimiento al que he aludido no se debe a una falta de popularidad, muy al contrario, sea cual sea la respuesta que obtengamos cuando decimos que somos antropólogos suele ser muy positiva. Practicamos una disciplina catalogada como "muy bonita", sin embargo, si nuestra conversación continúa, la siguiente pregunta suele ser tan temible como reveladora: ¿Para qué sirve eso?

No hace falta ser antropólogo para darse cuenta que "bonita" en este contexto significa "ornamental", es decir, un lujo de la sociedad. Una lectura menos misericorde justifica nuestra actividad por la vieja idea de que en este mundo "tiene que haber de todo". Convertidos así en una suerte de extraña horda que convive y merodea en su territorio sólo nos queda recurrir al argumento de la "utilidad" social si se quiere ganar una salida airosa y recuperar un lugar en la sociedad de mercado a la que, supuestamente, todos pertenecemos. Dicha conversación suele generar entre

1 Este artículo es una segunda versión de un texto que preparé para una oposición a una plaza de científico titular de antropología social del Departamento de Antropología de España y América del Instituto de Filología del CSIC. Los comentarios que suscitó el texto original, hoy corregido, me indujeron a redactar esta segunda versión que se beneficia de la crítica e interesantes matizaciones que hicieron Carmen Ortiz y Margarita del Olmo. No obstante, ninguna de ellas es responsable del resultado final. 
antropólogos una sensación de "choque cultural" en casa. Ante esta experiencia se añora, a veces, la "inocente" pregunta del trabajo de campo, cuando los nativos quieren saber qué es lo que el antropólogo quiere.

Sin embargo, al narrar esta anécdota que considero muy ilustrativa del estado actual de la antropología, no quiero destacar solamente el ambiguo estatus de que goza nuestra disciplina, sino mostrar cómo esa posición proviene de nuestros propios cuestionamientos y de las actitudes que hemos venido adoptando ante los cambios del mundo actual.

Mientras que, por un lado, la antropología disfruta de una posición académica e institucional no hace mucho inimaginable, por el otro hemos abierto la caja de Pandora. Según muchos, que tiñen sus argumentos de una retórica mesiánica como la de los movimientos homónimos que analizamos, sufrimos una crisis sin precedentes, apocalíptica. Según otros, estamos sujetos a una fortísima transformación. Con distintas valoraciones y en distintos grados, casi todos los antropólogos parecen añorar ese tiempo mítico en el que existían los maestros. Quedan algunos grandes personajes que tienden a ser considerados por nosotros más como "supervivientes" de un pasado mejor que como los líderes de las tendencias de pensamiento antropológico actual. Nos encontramos, como Geertz relata en sus memorias ${ }^{2}$, con la sensación de haber llegado demasiado pronto o demasiado tarde, de que el cambio en el que vivimos no es como un desfile en el que somos espectadores puesto que ya no es posible mantenernos fuera del objeto de estudio, y porque carecemos de una posición estática desde la que observar el cambio del mundo o de nuestra propia disciplina. Las fronteras que antes nos permitían mantener un orden, conectar las unidades discretas del parentesco, la estratificación social, los sistemas económicos, el mundo de valores, se han disuelto, y el orden y conexión que podemos dar a los fenómenos observados se nos aparecen frágiles y contingentes.

Con todo, tras esta introducción que parece el preámbulo a una declaración de derrota, quiero abordar el estado de la cuestión de la antropología con tono positivo ya que, en mi opinión, gran parte de los problemas y perplejidades que he señalado son propios de su vitalidad y de su considerable desarrollo, de su conexión con el mundo y una muestra, a su vez, de las líneas de evolución actual y futura.

Propongo, en el primer caso, aproximarnos al "canon" de la antropología actual revisando la evolución de un par de manuales de antropología que he seleccionado por su relevancia. Al modo del arqueólogo, rea-

2 C. GEERTZ, After the Fact. Two Countries, Four Decades, One Anthropologist (Cambridge, Mass.: Harvard University Press, 1995). 
lizaremos dos cortes temporales para averiguar qué permanece y qué ha cambiado en los últimos años.

Con respecto al ejercicio de la antropología me centraré en tres congresos de reconocido prestigio: la primera Conferencia de la Asociación Europea de Antropólogos Sociales (EASA) que se celebró en Coimbra, Portugal, en el verano de 1990 (de cuyas sesiones se han publicado seis colecciones de textos en la serie de antropología social de la EASA en la editorial Routledge); la reunión anual de la Asociación de Antropología Americana, que se desarrolló en Washington, DC, en noviembre de 1997 y el 14 Congreso Internacional de la Unión Internacional de Ciencias Antropológicas y Etnológicas que se celebró durante Julio de 1998 en Williamsburg, Virginia. Añadiré, para terminar, una serie de comentarios sobre la antropología en España.

\section{DE LOS MANUALES}

Una simple ojeada a un manual de antropología reciente, sobre todo si recordamos aquellos que estudiamos al final de los años 70 , nos puede ofrecer una clara idea de cuáles son las nuevas coordenadas en las que se ubica la disciplina y los nuevos temas que atraen la atención de los antropólogos. Nos muestra, asimismo, un modo diferente de ejercer la disciplina.

En un primer vistazo se constata que el término "antropología" se suele asociar a "contemporánea" ${ }^{3}$, comienza a aparecer este último en los

3 Este comentario, no sólo se fundamenta en los textos a los que voy a hacer referencia, sino en otros muchos manuales que he revisado con el objeto de seleccionar dos significativos para desarrollar los argumentos que siguen. Además de los textos que menciono a lo largo del texto, revisé los siguientes: Jesús Azcona, Para comprender la antropología (Estella, Navarra: Editorial Verbo Divino, 1991); Victor BARNOuw, Ethnology. An Introduction to Antropology, Volume Two (Chicago: The Dorset Press, 1987 [5ta ed.; 1 ed. 1971]); Carol R. EMBER y Melvin EMBER, Cultural Anthropology (Englewood Cliffs, NJ: Prentice Hall, 1988 [5ta. ed.]); Michael C. HowarD, Contemporary Cultural Anthropology (Boston, Mass.: Little, Brown and Company, 1986 [2da ed; 1 ed. 1983]); James PeOPLES y Garrick BAILEY, Humanity. An Introduction to Cultural Anthropology (St. Paul, MN: West Publishing Company, 1988); Thomas RHYs Williams, Cultural Anthropology (Englewood Cliffs, NJ: Prentice Hall, 1990), y Emily A. SCHULTZ y Robert H. Lavenda, Cultural Anthropology. A Perspective on the Human Condition (St. Paul, MN: West Publishing Company). Si exceptuamos el antiguo "superventas" de Marvin Harris, he considerado innecesario incluir manuales "más clásicos" como los de E. A. Hoebel, R. Linton, M. Herskovits, J. Beattie, C. Kluckhohn, S. F. Nadel, o R. Beals y $\mathrm{H}$. Hoijer. 
encabezamientos de los capítulos e incluso en los títulos de los propios manuales, como una declaración explícita por parte de sus redactores de una voluntad de acercarse al mundo actual. Occidente y las llamadas sociedades complejas son ya materia antropológica en sí mismas, y no el lugar privilegiado a partir del que los antropólogos construyen su relativismo cultural. El tiempo en el que "nuestro mundo" era usado a modo de contraste parece desvanecerse, y con él, ese territorio imaginario de fronteras bien definidas que el antropólogo Bernard S. Cohn denominó "Antropologilandia" 4 .

En este "país", surgido de la consolidación en el mundo académico de la antropología, los nativos que eran, sin lugar a dudas, "ellos" u "otros" se presentaban como "auténticos", sin contaminar por las fuerzas corrosivas del Occidente avasallador. A menudo, las etnografías nos brindaban una imagen primigenia del pueblo estudiado, antes de que el inexorable paso del tiempo y la llegada del misionero y del comerciante alterara definitivamente este espacio sin tiempo, idílico en algunos casos. Resulta curioso constatar cómo esta etnografía de salvamento, una vez reivindicada su urgencia, olvidaba los hechos de la propia historia nativa y de su creciente conexión con un mundo exterior que se redefinían día a día. Cuando la ficción de los etnógrafos de la insularidad de los nativos con respecto al mundo con "tiempo" ya no podía mantenerse más, una nueva generación de antropólogos reivindicaba su capacidad de acceder a las estructuras simbólicas y socio-culturales de los nativos y consolidaba antropologilandia. Con ellos, con esos nativos en esas etnografías, la antropología culminaba un periodo de abstracción y construcción teórica en la que el hombre, la sociedad y la cultura estudiados perdían, primero el tiempo y luego, incluso, el espacio, ocupado ya por los colonizadores o, más indirectamente, por las fuerzas modernizadoras y de globalización.

Desde el punto de vista teórico, son múltiples las razones y justificaciones que, en muchos casos muy legítimamente, podemos aducir para explicar el estado al que había llegado la antropología general, la etnografía o la propia teoría antropológica general. Sin lugar a dudas las señas de identidad de la antropología que se consolidó en los años treinta tras la primera crisis del imperialismo occidental procedían, por un lado, de una antropología que buscaba su modelo y se originaba inspirada en la ciencias sociales, mientras que, por otro, se consolidaba una escuela de pensamiento teórico social surgido de expertos de biblioteca formados

4 "History and Anthropology: The State of the Play", An Anthropologist among the Historians and Other Essays (Delhi, India: Oxford University Press, 1990), p. 19. 
en el derecho 5 . Unos años después, tras la Segunda Guerra Mundial y la conquista de la independencia de las naciones no occidentales en los años 60 , se hizo imposible mantener este viejo paradigma que era capaz de crear una doble ficción: mientras que la distinción entre el objeto de estudio y quien lo estudiaba era clara, éste último era, a su vez, capaz de llevar a cabo una observación participante que le permitía acceder a un conocimiento de primera mano de la vida cotidiana de sus "nativos", así como de sus valores. Gracias a ellos, en un estadio ulterior de trabajo, era también capaz de revelar los códigos ocultos, sus "motores íntimos" e integrar esos "descubrimientos" en un corpus teórico impulsado por tres "ismos" o principios fundamentales: holismo, comparativismo y relativismo.

¿Cómo armonizar el considerable cuerpo teórico antropológico con el estudio de unas sociedades que habían perdido su inocencia "salvaje" o "natural", a las que era imposible abarcar con los útiles tradicionales de la antropología y, sobre todo, con el contacto directo del trabajo de campo? Los nativos son ahora ciudadanos, como dice Geertz en una reseña recién aparecida, y los primitivos minorías ${ }^{6}$.

Si retomamos esos manuales que ostentan las palabras "contemporáneo", "global", "sistema mundial" o "fuerzas modernizadoras" en sus textos, por citar sólo algunos de los ejemplos más significativos, parece claro, ya desde sus propios índices, que la compartimentalización clásica de parentesco, organización social y política, adaptaciones económicas, lenguaje, religión o magia, ha cedido el paso a una no muy distinta organización de los epígrafes que muestra, sin embargo, concepciones substancialmente nuevas. De modo gradual hemos pasado de manuales como el de Marvin Harris $^{7}$, que actualizaba su cuarta edición mediante la inclusión de dos nuevos capítulos dedicados al mundo que nos esperaba allí fuera (me refiero a los capítulos sobre "pobreza y cultura" y "subdesarrollo y cambio culturaln), además de una sección de estudios modernos y occidentales (el capítulo de economía política del estado), a otro tipo de manual mucho más reciente, como la sexta edición del texto de Conrad Phillip Kottak,

5 Muchos de los "primeros" antropólogos, como Tylor en el Reino Unido o Morgan en los Estados Unidos eran abogados o habían estudiado derecho inicialmente. Su conceptualización del mundo "primitivo" estaba profundamente influida por el derecho, como en mi opinión indicó oportunamente Adam KuPER en The Invention of Primitive Society. Transformations of an Illusion (Londres: Routledge, 1988).

6 "Deep hanging out", The New York Review of Books, XLV, 16 (22 de octubre de 1998), p. 69.

7 Introducción a la antropología general (Madrid: Alianza, 1987). La primera edición estadounidense data de 1971. 
publicada en España en $1994^{8}$ con añadidos de ocho antropólogos españoles (M." Jesús Buxó, Tomás Calvo Buezas, Juan A. Fernández de Rota, Francisco Giner Abati, José A. González Alcantud, Dolores Juliano, Beatriz Moncó y Paz Moreno), en el que no sólo aparecen nuevos capítulos sobre los temas que generan un mayor interés hoy, sino que, sobre todo, se aplica una sola lente unificadora al mundo en el que habitamos. Así, por ejemplo, las sociedades recolectoras, se asemejan notablemente en su flexibilidad a las modernas sociedades occidentales. Poco a poco, la inclusión de la sociedad occidental, y la restauración de las dimensiones espacial y temporal en el análisis antropológico, han conseguido empezar a hacer despuntar una nueva imagen del mundo.

Kottak, a diferencia de otros manuales clásicos adopta una perspectiva más globalizadora e introduce una serie de capítulos relativamente novedosos, como son los dedicados a "etnicidad y relaciones interénticas", "Construcción cultural de la raza", "sistema mundial, industrialismo y estratificación", "género", "antropología aplicada", "colonialismo y desarrollo", "intercambio cultural y supervivencia" y "cultura popular estadounidense"; al que los autores españoles añaden secciones sobre las "culturas en España", "racismo en España", "raíces biológicas del comportamiento humano y de la cultura (Etología humana)", "adaptación y economía" "clientelismo social y político en las sociedades mediterráneas", "mujer y familia en España y América", "Cosmología popular gallega" o "prospectiva antropológica".

De poco vale preguntarnos, como dice Geertz en sus memorias, qué es lo que ha cambiado, si el mundo o nosotros mismos. Lo cierto es que las transformaciones que acabo de esbozar son de mucha mayor magnitud de lo que se puede destacar tras una revisión de los dos manuales seleccionados. De hecho, estas obras tienden a recoger y transmitir aquellos conocimientos aceptados casi unánimemente por la comunidad antropológica. Muestran más la gradual transformación de la disciplina, que las grandes revoluciones o rupturas que algunas escuelas o colegas han pregonado. Desde el punto de vista del desarrollo de la antropología, considero que los manuales representan una tradición de nuestra historia, que enfatiza, como es lógico, el continuismo y, como pretendo mostrar al abordar el ejercicio de la profesión, que se ajusta en mayor medida al mundo real que pueblan los antropólogos. Muchos de los cataclismos que han proclamado los estudiosos del cambio son más el fruto de una nueva percepción de nuestro entorno y de la rebelión de los estudiados, que el cambio de Era al que tanto aluden.

8 Antropología. Una exploración de la diversidad humana con temas de la cultura bispana (Madrid: McGraw-Hill, 1994). 


\section{DEL CAMINO RECORRIDO}

Hablaba hace poco de las técnicas de eliminación del tiempo e, incluso, del espacio, que ciertas escuelas antropológicas desarrollaron en la primera mitad del siglo $\mathrm{xx}$. Las sociedades eran o simples y reductibles a una monografía de varios cientos de páginas, o complejas, como la nuestra, e irreductibles, a menos que hiciéramos uso de métodos, conceptos o definiones tales como "red" o "subcultura de la pobreza", que nos permitiesen aislar unidades de análisis mucho más pequeñas y con menos variables.

Pese a dichas técnicas, que en modo alguno eliminaban de nuestra vista la compleja intersección de tendencias y realidades que plagan el estudio, era prácticamente imposible ya la construcción de la ficción de un "cuasi-laboratorio" como en el que trabajaron Malinowski o RadcliffeBrown. La antigua clave de nuestra disciplina, el trabajo de campo, ha sufrido también una profunda transformación, los nativos no sólo leen o contestan nuestras monografías sino que nuestra entrada en sus comunidades se ve sujeta a una compleja negociación. Por si ello fuera poco, el propio trabajo de campo, hasta hace poco patrimonio de la antropología, comienza a convertirse en punto de referencia de otras disciplinas a las que fascina su metodología cualitativa.

Por ello, su pariente cercano, la etnografía, se ha convertido no sólo en una actividad practicada por antropólogos en comunidades reducidas, sino, también, en nuestros propios entornos, y por otras disciplinas, entre las que podemos mencionar la sociología, la geografía, los trabajos sociales o de urbanismo, el marketing, la historia de la ciencia, el estudio de los ámbitos laborales o, sobre todos, el periodismo, por mencionar sólo algunos de los más destacados.

Ahora bien, dicha popularización o ampliación de los mundos a los . que puede accederse mediante la antropología, lleva aparejada una profunda y radical transformación, que sólo puede comenzar a revelarse mediante un análisis más meticuloso y profundo de los manuales, y continuarse en la literatura antropológica más reciente.

La etnografía atraviesa hoy, como acertadamente señalan George E. Marcus y Michael M. J. Fischer ${ }^{\text {, }}$, un momento de escritura experimental semejante al que se vivió en los años 20 y 30 de nuestro siglo, cuando se estaba tratando de desarrollar nuevas técnicas de descripción de la

9 Anthropology as Cultural Critique. An Experimental Moment in the Human Sciences (Chicago: University of Chicago Press, 1986). 
realidad. Esa consciencia, que ya mencioné, de las limitaciones de nuestra metodología y de las dificultades que las evidenciaron, ha provocado el florecimiento de nuevas tendencias en el estudio antropológico y la recuperación de la función crítica, que hace algunas décadas caracterizó a la disciplina. La experimentación, más activa en la búsqueda de nuevas formas de expresión escrita, visual o "multimedia", adecuadas a las nuevas posiciones teóricas, parece haber ganado un lugar relevante en el panorama antropológico frente al simple "oficio" de nuestro trabajo.

La renovación del movimiento experimental no debe considerarse, en mi opinión, como un fenómeno gratuito, o un lujo de una disciplina sólidamente acomodada en la academia. Aunque algo de ello pueda deberse a esa consolidación y a otros factores más o menos fortuitos, es notorio observar cómo se asocian la experimentación y la vanguardia con periodos de fuerte consciencia de las transformaciones mundiales. Dicha consciencia del cambio, del notorio empequeñecimiento del mundo, ha hecho, por un lado, más difícil escribir etnografía y, por otro, más deseable o necesario abordarla de un modo más abierto y experimental - como, sin duda, ya lo fue hace sesenta o setenta años. Ahora bien, recurrir al argumento de la necesidad de una representación más adecuada del mundo, para explicar el giro hacia la etnografía experimental, me parece claramente insuficiente. Bajo esta perspectiva es posible que hubiera bastado con algo parecido a las reformas que manuales como el de Marvin Harris introdujeron en los años 80, es decir, a nuevos capítulos sobre el subdesarrollo o la pobreza, que "actualizasen" nuestros objetos de estudio.

Sin embargo, la etnografía experimental que ahora convive con las tendencias clásicas en la academia, no hubiera alcanzado la difusión y el impacto que hoy tiene si no hubiera sido por el desarrollo de nuevas tendencias teóricas cuyo origen puede rastrearse, al menos, hasta los años 60. Entonces compartieron sus impulsos renovadores con los expertos dedicados a adaptar los modelos de la lingüística a la teoría antropológica. En las décadas siguientes, las de los 70 y 80 , dichos modelos dieron paso a los procedentes de la crítica e interpretación literarias y se asistió a un periodo, todavía no cerrado, en el que los teóricos sociales y culturales tendieron a confluir con aquellos practicantes de la etnografía que estaban preocupados por representar mejor el mundo nooccidental. De algún modo, la autocrítica hacia nuestro modo de representación y nuestra realidad, se constituyeron en el punto de encuentro entre los teóricos y los etnógrafos.

La búsqueda de nuevas dimensiones en la escritura etnográfica era producto, primero del reconocimiento de la necesidad de una representación del "Otro" más adecuada a la imagen que ese "otro" tenía de sí mis- 
mo, segundo, de la adecuación al mundo postcolonial y, tercero, del descubrimiento del papel del antropólogo como autor de mundos literarios a través de la escritura. El tema de la representación de la realidad, más que el de la descripción, ocupó una posición central en la reflexión antropológica, hasta el punto de que mucha de la literatura actual de la disciplina se parece más al género ensayístico que a las monografías clásicas. Las propias representaciones son hechos sociales, como reclamaba un conocido artículo de Paul Rabinow de $1986^{10}$. El texto cobraba un nuevo significado y dimensión para el antropólogo, y perdía su calidad de espejo o retrato de una realidad para convertirse en realidad en sí misma. Esa dimensión provocaba, a su vez, que el autor, como Alicia en el país de las maravillas, cayera en la madriguera que da entrada al mundo de lo maravilloso o absurdo y se viera al otro lado del espejo, incorporado al texto que él mismo había creado. Muerto en estas circunstancias el género realista en antropología, se imponía el ensayo, y el autor reflexionaba acerca de sí mismo ganando consciencia de su propio papel en el proceso de investigación. El antropólogo creaba como autor y representaba, a la vez, un mundo del que también era y se reconocía como parte integrante. El estilo de redacción, algo que caracterizaba la prosa de los antropólogos más influyentes, cobraba asimismo un nuevo protagonismo, y se hacía evidente su valor para conferir verosimilitud al relato ${ }^{11}$

Sin duda, todas estas innovaciones muestran el camino recorrido por la antropología desde la época gloriosa de los grandes trabajos de campo y monografías que crearon no sólo nuestra imagen de algunas sociedades y culturas de África, Asia, Oceanía o América, sino también, muchos de los fundamentos de la teoría social y cultural. Llegar a la isla de Tikopia era para Raymond Firth una experiencia iniciática que el etnógrafo compartía en clave poética con el lector para, con sus descripciones, mostrarnos el complejo entramado de una sociedad aborigen alejada del vértigo

10 "Representations are Social Facts: Modernity and Post-Modernity in Anthropology", Essays in the Anthropology of Reason (Princeton, NJ: Princeton University Press, 1996). Me permito la libertad de indicar como autoridad a Rabinow, en vez de al "clásico" Foucault, por dos razones, en primer lugar por una formal: se trata de una excelente síntesis adaptada a la antropología de algunas de las claves analíticas del pensador francés. En segundo, por ser Rabinow un buen ejemplo antropológico de la fusión de varias líneas del pensamiento postestructuralista francés (Barthes, Derrida, Foucault...), y el editor de las obras de Foucault en inglés.

11 Es bien conocido el libro de C. GEerTZ, El antropólogo como autor (Barcelona: Gedisa, 1989) [Works and Lifes. The Anthropologist as Author (Stanford, Calif.: Stanford University Press, 1983)]. 
al que estaba sometida la sociedad occidental del periodo de entreguerras. Firth, en el primer capítulo, titulado "En la Polinesia primitivan (él mismo procedía de la polinesia no-primitiva, es decir, de Nueva Zelanda), abría el libro con la siguiente descripción:

En el fresco de la primera mañana, momentos antes del amanecer, la proa del Southern Cross se dirigía hacia el horizonte, hacia el este en el que era apenas visible un diminuto perfil azul oscuro. Lentamente creció hasta convertirse en una escabrosa masa montañosa que se erguía, vertical, sobre el océano. Luego, conforme nos aproximamos a unas millas de ella, apareció en torno a ella un estrecho anillo de tierra baja y plana de densa vegetación. El plomizo y gris día, con sus nubes bajas, acentuó la mala impresión que tenía de un pico solitario, salvaje y tormentoso, empujado hacía arriba en la inmensidad de las aguas circundantes ${ }^{12}$.

Gracias a esos textos clásicos, considerados de un modo un tanto superficial como puramente empíricos, comenzamos a obtener las herramientas y tramas que nos permitieron abordar una comprensión holística, comparada y relativista del hombre. Del mismo modo que ellos abrieron un camino, considero que este nuevo espíritu experimental no debe minusvalorarse en conjunto, o como hacen algunos críticos, englobarse bajo el epíteto de "postmoderno". De hecho, muchos de los que exploran las dimensiones a las que he aludido se sentirian claramente ofendidos si se les considerara, sin más, postmodernos, ya que conciben su obra como una reflexión sobre la modernidad. En todo caso, si seguimos al sociólogo Anthony Giddens ${ }^{13}$, el perfil de la modernidad actual, si bien puede augurar una nueva era, no es más que una consecuencia o exacerbación de la modernidad. No obstante, dicha perspectiva sociológica de Giddens no entra, en mi opinión, en contradicción con el pensamiento postmoderno propiamente dicho, si consideramos a éste como a un proyecto intelectual que pretende reubicar la cultura occidental dentro de un marco mayor de índole postcolonial.

El otro ámbito en el que los antropólogos han aportado más a la renovación y desarrollo de su disciplina, ha sido, paradójicamente, el del estudio del pasado de la disciplina. La historia de la antropología ha sido y es, sin duda, uno de los campos que ha colaborado más decisivamente a abordar el trabajo de un modo más crítico e inquisitivo, no sólo en el mundo que habitamos, sino sobre todo en nosotros mismos. Estudiando

12 We, the Tikopia. A Sociological Study of Kinship in Primitive Polynesia (Stanford, Cal.: Stanford University Press, $1983\left[1 .^{a}\right.$ ed. 1936]).

13 Consecuencias de la modernidad (Madrid: Alianza, 1993). 
a Durkheim o Evans-Pritchard, a Boas o Morgan somos más conscientes del modo en el que se operaba en el taller del antropólogo, de cómo los personajes, sus historias y contextos conformaban la genialidad indiscutible y la creatividad de los precursores. No se puede afirmar sin más, sin embargo, que aquellos que miran al pasado hayan revolucionado el actual pensamiento antropológico. A menudo su reconstrucción del mundo que habitaron nuestros antepasados disciplinares ha servido para revelar, sin pretenderlo, sus debilidades o de piedra de toque para aquellos que pretendían recurrir, con las herramientas de hoy, a las teorías y estrategias de hace algunas décadas.

El caso del antropólogo Adam Kuper, primer presidente de la Asociación Europea de Antropólogos Sociales, es muy representativo del tipo de aportaciones al que me estoy refiriendo. Adam Kuper, que se inició en la antropología dentro de la línea tradicional e iniciática del trabajo de campo, pasó a hacer un análisis histórico de la evolución de la antropología social británica entre los años 1922 y 1974. Antropología y antropólogos ${ }^{14}$ es, sin duda, el preámbulo a su mucho más reciente y estimulante monografía sobre la invención de la sociedad primitiva ${ }^{15}$ en la que vemos de qué modo una visión de la sociedad como primitiva o distante de la nuestra, tiene su origen en un grupo de intelectuales cuyo común denominador o influencia determinante es el derecho. Nos muestra, asimismo, cómo esas primeras formulaciones teóricas sobre los conceptos de sociedad y otros asociados a la descripción y análisis de esa sociedad han sobrevivido gracias a las transformaciones de esa ilusión. Su posición, distinta a la de aquellos que han iniciado su reconversión metodológica y teórica abordando la crítica cultural y la concepción del mundo como si se tratara de un texto, es mucho más teórica a la "antigua usanza". Frente al "giro cultural ${ }^{16}$ que se ha producido en el pensamiento occidental de las últimas décadas, adopta una alternativa más parsimoniosa y reformista y no duda en contraatacar con una crítica de la cultura tal como ha sido defendida por antropólogos como Geertz, David Schneider o Marshall Sahlins ${ }^{17}$.

14 (Barcelona: Anagrama, 1973).

15 Me refiero a la ya citada: The Invention of Primitive Society. Transformations of an Illusion (Londres: Routledge, 1988).

16 Hago uso aquí del título de una reciente selección de textos de Fredric JamesON, The Cultural Turn. Selected Writings on the Postmodern, 1983-1998 (Londres: Verso, 1998).

17 Véase Adam KuPER, Culture. The Antbropologists'Account (Cambridge, Mas.: Harvard University Press, 1999). 
Puede parecer, siguiendo mis propios argumentos, que la teoría ha jugado un papel subordinado o, incluso, secundario dentro del panorama que estoy dibujando, y que, si existe teoría social en antropología no lo es dentro de lo que tradicionalmente se denomina antropología cultural. Es decir, las escuelas británicas asociadas bajo el título de "antropología social" y las estadounidenses, que lo hicieron con el de "antropología cultural", llevaron a cabo un reparto de áreas de estudio en el que la primera pensaba en lo social mientras que la segunda lo hacía en lo cultural. Si bien, no se puede negar un énfasis distinto en la relevancia de la dimensión social o cultural, dicha "división" parece legitimar más un encasillamiento excesivamente formalista que el curso real del pensamiento antropológico.

Comenzaré por la última de las cuestiones a las que he hecho referencia, es decir, la de haber sesgado mi discurso hacia la antropología cultural. Aunque de momento sólo ofreceré una respuesta parcial y algo coyuntural, intentaré aclarar lo que, en mi opinión, es una visión excesivamente simplista de la llamada antropología cultural estadounidense y de la profunda orientación social de algunas de las escuelas y tendencias teóricas más importantes de ese país. Por otro lado, la enseñanza habitual de los antropólogos británicos y algunos franceses en los Estados Unidos, hace difícil creer en una antropología cultural aislada de las contribuciones británicas y francesas en teoría social. Por ejemplo, RadcliffeBrown, llegado a la Universidad de Chicago en los años 30, ayudó al Departamento de Antropología a consolidar una identidad diferenciada del influyente Departamento de Sociología, así como conferir a los antropólogos un substrato teórico de corte social del que carecía la escuela de Chicago de Etnografía Urbana nacida en el Departamento de Sociología. Si bien ambas consolidaron la etnografía como su herramienta fundamental de trabajo, la antropología estadounidense incorporó como un elemento relevante la reflexión social. La aparición de antropólogos como Fred Eggan ${ }^{18}$, Robert Redfield (quien introdujo el libro anterior y abordó de modo influyente las distintas características de la sociedad "folk" y urbana) o Edward Spicer ${ }^{19}$, así como otros muchos que se formaron imbuidos por estas ideas, es un buen reflejo de ello. En alguna medida, un rasgo relevante de una parte de la antropología estadounidense hoy es su integración en un solo análisis de las dimensiones social y cultural.

18 Social Organization of the Western Pueblos (Chicago: Chicago University Press, 1950), o que editó Social Anthropology of North American Tribes (Chicago: Chicago University Press, 1955).

19 Cycles of Conquest. The Impact of Spain, Mexico, and the United States on the Indians of the Southwest, 1533-1960 (Tucson, Az.: Arizona University Press, 1962). 
De hecho, todavía hoy, algunos departamentos influyentes, como el de la Universidad de Harvard, conservan el adjetivo de antropología social y cultural en sus programas e incluyen entre su profesorado una relevante nómina de profesionales británicos o antropólogos con formación de esas islas, como son David Maybury-Lewis, los discípulos de Edmund Leach, Stanley J. Tambiah y Nur Yalman, o el renovador Michael Herzfeld. También numerosos manuales, como el ya mencionado de Kottak, consideran que el término antropología cultural es, en realidad, un modo de abreviar "Socio-cultural" ${ }^{20}$.

Si atendemos, por otra parte, a las definiciones que se emplean de sociedad y cultura en el mismo manual, se hace evidente la incapacidad de que la cultura sea algo separado de la sociedad, como defendió Leslie White en su tiempo ${ }^{21}$.

Por otra parte, las insularidades británica, francesa y estadounidense en lo académico no son tan acentuadas como para haber impedido un fructífero intercambio en la esfera de la teoría socio-cultural. Así, personajes británicos actuales como Adam Kuper o Mary Douglas residen o visitan continuamente los Estados Unidos, otros, ahora asentados en ese país como Michael Herzfeld visitan frecuentemente Europa y están al tanto de la actividad en Francia y en el Reino Unido; y franceses como Pierre Bourdieu o antropólogos sociales escandinavos como Fredrik Barth o Ulf Hannerz dividen su tiempo en ambos mundos y nos recuerdan el intercambio intercontinental que personajes como Foucault, Jacques Derrida, Radcliffe-Brown o Evans-Pritchard realizaron en su día. Este flujo y reflujo que se centra en torno a algunas instituciones claves de los tres países, ha ayudado a que antropólogos sociales y culturales, o socio-culturales, hayan combinado sus distintas perspectivas y énfasis en torno a las tendencias más influyentes en la actualidad. De algún modo, como hace tres años Fredrik Barth decía en una charla en el Massachusetts Institute of Technology (M.I.T., Cambridge), él se sentía más identificado con el colectivo internacional de antropólogos como comunidad, que con la mayor parte de sus compatriotas con los que compartía un universo muy poco coincidente. Por el momento, esta identificación con un no espacio, o "transespacialidad", si me permiten el término, es una de las características más destacadas de la actualidad ${ }^{22}$.

\footnotetext{
20 Éste es el tipo de antropología que se puede encontrar en la mayoría de los grandes departamentos de las universidades de los Estados Unidos. Las otras áreas reconocidas allí son, además de la socio-cultural, la arqueológica, la biológica y la lingüística.

21 Véase El concepto de cultura: textos fundamentales, editado por J. S. Kahn (Barcelona: Anagrama, 1975).

22 Véase, por ejemplo, la introducción a Homi K. BHABHA, The Location of Culture (Londres: Routledge, 1994).
} 
Poco a poco, desde los comentarios que dieron inicio a mi artículo, he ido esbozando las principales características de la antropología más reciente, sus orígenes y algunas de las líneas de evolución que hasta ahora han ido imponiéndose, y que parece que siguen abiertas y dispuestas a brindarnos nuevas fronteras de análisis. Visto desde otra perspectiva, pudiera dar la sensación de que nuestros "avances" son más una "disolución" de los viejos y sólidos fundamentos de la antropología que tantos años ha costado establecer y generalizar. En cierto modo sí podemos hablar de "disolución" de teorías y conceptos ante el acoso de la crítica de nuevas generaciones de antropólogos y el nuevo contexto mundial en el que habitamos. Sin embargo, la "disolución" a la que me refiero no es más, en mi opinión, que el tejer y destejer de una disciplina muy activa. El renacimiento de la vocación experimental, junto con las nuevas tendencias críticas y escuelas en boga más o menos consolidadas, no han afectado sólo a la propia escritura de la antropología, al papel del antropólogo, o a la plena inclusión de Occidente en su ámbito de estudio. Como cabría esperar, la propia teoría central de la antropología social ha estado sujeta, y todavía lo está, a una intensa reconsideración. Ya cuando hablé de la obra de Kuper aludí a cómo éste consideraba el concepto de sociedad primitiva como una ilusión que había sido capaz de transformarse con el tiempo. ¿Se ha mantenido esta ilusión? O ¿se ha reformulado el concepto de sociedad?

\section{DEL EJERCICIO}

Con esta cuestión llegamos ya a la sección que anuncié sobre el ejercicio de la antropología ya que, con la evolución que ha sufrido nuestra disciplina, parece difícil desarrollar cuestiones teóricas de cualquier nivel sin que las mismas se ajusten a los casos de estudio de los que surgen y en los que se han desarrollado.

Desde al menos los años setenta, el concepto de sociedad y estructura social han sido denunciados como inadecuados e insatisfactorios para el trabajo de campo, una adecuada comprensión de las sociedades estudiadas necesita de una comprensión dinámica de la sociedad. La monografía de Renato Rosaldo sobre los Ilongot de Filipinas, publicada en $1980^{23}$, es una excelente muestra de esa crítica a los conceptos de organización social y sociedad en un marco de análisis sincrónico. "Mi opi-

23 Ilongot Headbunting, 1883-1974. A Study in Society and History (Stanford, Ca.: Stanford University Press, 1980). 
nión — decía Rosaldo- es que el método analítico de congelación del tiempo con el objetivo de comprender mejor las relaciones estructuralfuncionales del todo social ha producido en sí mismo la ilusión de unos "primitivos sin tiempo" ${ }^{24}$, así como ignorar la historia como proceso estudiado por los antropólogos o, incluso, la propia consciencia que sus actores tienen de ella.

Un número creciente de ponencias, artículos y trabajos muestran cómo esa dificultad puede soslayarse en muchos casos y temas que en la actualidad merecen la atención de los expertos. Sin embargo, en muchos otros, combinar el paso del tiempo con los múltiples elementos necesarios para el análisis de los grupos estudiados, hace muy difícil mantener la idea de sociedad como un conjunto de relaciones relativamente cerradas y homogéneas entre humanos.

No considero casual que la primera conferencia de la recién fundada Asociación Europea de Antropólogos Sociales (EASA), celebrada en Coimbra, Portugal, durante el verano de 1990, abordara una reflexión teórica sobre la sociedad, el modo de conceptualizarla, cómo otras culturas piensan sociológicamente, si es así que lo hacen, o en qué consiste la sociedad civil desde una perspectiva comparada y teórica.

En el marco de una afirmación de la emergente nueva identidad disciplinar europea, preocupada por distinguirse de otras, gracias a su componente "social", se puede constatar el rechazo de una fórmula única para abordar la comprensión de la sociedad y el acuerdo en un proyecto común, pero diverso, de reconciliar la visión de actor y observador de la sociedad; así como reivindicar la capacidad de la etnografía, frente a la demoledora crítica hecha por los pensadores "postmodernos" americanos, para ofrecer datos y resultados que permitan hacer uso de distintas tramas teóricas y construir argumentos de análisis transcultural. Es posible que estas afirmaciones, que hizo Kuper como primer presidente de la Asociación Europea de Antropólogos Sociales, sean un modo particularmente operativo para fijar una demarcación clara entre los cada vez más inestables campos de la antropología social y cultural, más que una manifestación del nuevo nacionalismo europeo que mencionaba, o el deseo de diferenciarse de la órbita estadounidense.

El concepto de sociedad, tal como lo perfiló Durkheim cuando reclamó una sociedad y una cultura para cada persona, se ha disuelto y, sin embargo, el mismo concepto, aunque transformado, sigue siendo central hoy. Mientras que para Ulf Hannerz ese tipo de sociedad cerrada ha desapareci-

${ }^{24}$ Op. cit., p. 27. 
do, engullida por el influjo de los procesos sociales globales ${ }^{25}$, para Fredrik Barth el concepto durkheimiano de sociedad ha sido simplemente una fantasía europea ${ }^{26}$. Las sociedades, dice este autor, han existido siempre, son desordenadas y se encuentran en cualquier lugar. Ahora bien, allí donde Hannerz y Barth parecen reivindicar un enfoque más renovador, Daniel de Coppet ${ }^{27}$ cree más en una concepción sociológica tradicional, en la que valores y actos se integran y constituyen la unidad de análisis social (como reafirmación de valores). En suma, ahora volviendo a Barth, es necesario considerar la sociedad como el contexto de acciones y resultados de acciones" ${ }^{28}$ no como una cosa en sí misma; sólo así es posible, tal como yo lo entiendo, integrar tanto la visión del observado y del observador, como de los grupos más simples incluidos en sistemas mayores o las diversificadas sociedades urbanas actuales ¿No es acaso esta perspectiva de la sociedad una conceptualización más acorde con las coordenadas básicas de la antropología hoy?, ¿una que se sitúa más en el plano de la acción de los individuos, del flujo de información y de la interrelación en múltiples niveles?

Sin contradecir las diferencias entre antropología social europea y crítica postmoderna americana, entendida ésta en un sentido muy amplio, creo que una perspectiva más abstracta pone en sintonía ese modo distintivo de hacer europeo y muchos de los desarrollos característicamente estadounidenses que también he mencionado. Más complejo, dentro de este juego de oposiciones, es el lugar que ocupa la tradición francesa. Sin embargo, me gustaría destacar cómo la más influyente de las mentes galas dedicadas a la teoría social, Pierre Bourdieu, es un habitante tan presente en el mundo sociológico y antropológico francés como en el estadounidense ${ }^{29}$. Su sociología reflexiva ${ }^{30}$ es una manifestación de una

25 "The Global Ecumene as a Network of Networks", Conceptualizing Society, editado por Adam Kuper (Londres: Routledge, 1992).

26 "Towards Greater Naturalism in Conceptualizing Societies", Conceptualizing Society (Londres: Routledge, 1992).

27 “Comparison, a Universal for Anthropology: from 're-presentation' to comparison of hierarchies of values", Conceptualizing Society (Londres: Routledge, 1992).

28 Op. cit., p. 31.

29. En particular su constantemente citado Outline of a Theory of Practice (Cambridge: Cambridge University Press, 1977). Si menciono la edición inglesa es porque ésta es una edición actualizada por el propio autor de la primera que se publicó en francés en 1972, Esquisse d'une theorie de la pratique (Ginebra y Paris: Droz).

30 En 1994 George E. Marcus escribió un interesante artículo sobre las ideologías de la "reflexividad": "On Ideologies of Reflexivity in Contemporary Efforts to Remake the Human Sciences", Poetics Today 15, 3; reeditado en su libro Ethnography through Thick and Thin (Princeton, NJ: Princeton University Press, 1998). 
serie de desarrollos teóricos que, aunque conscientes del papel del autorinvestigador, le niegan la pertenencia al mundo estudiado gracias al dominio de una teoría distanciadora ${ }^{31}$. Su influyente aportación teórica se encuentra lejos, por lo tanto, de los criterios postmodernos (que admiten, por ejemplo, la alternativa de una relación dialógica o dialogada entre aquellos a los que estudiamos y nosotros); y, es, sin embargo, un notable avance con respecto a la falta de dinamismo de las perspectivas estructuralistas sin alejarse radicalmente de ellas. La tercera vía que representa Bourdieu es, según entiendo, un resultado semejante al que Kuper puede representar en Inglaterra, si bien este último se confina fundamentalmente en un ámbito de historia de la teoría antropológica.

Ahora bien, si dirigimos nuestra atención hacia el ejercicio en general de la antropología, podemos comprobar otros dos rasgos que considero relevantes. En primer lugar, el crecimiento y dinamismo de una antropología dedicada a ámbitos que podría denominar como tradicionales, y que continúa innovando de un modo que algunos denominan "ecléctico". Y, en segundo, la preocupación que nuestra disciplina muestra por su propio porvenir en un mundo que, como diría Edmund Leach, todavía sigue en explosión ${ }^{32}$.

Tanto la Reunión Anual número 96 de la Asociación de Antropología Americana, cuyo tema central fue: “Los futuros de la antropología: Práctica, imaginación y teoría en el siglo XXn"; como el decimocuarto Congreso Internacional de Ciencias Antropológicas y Etnológicas, cuyo lema fue: «El siglo XXI: El siglo de la antropología", centraban su atención en el futuro, fijando en su horizonte de trabajo el cercano cambio de siglo, y manifestaban su deseo de que el papel de la antropología fuera destacado a escala planetaria. Las similitudes en los programas generales eran grandes y mostraban una preocupación considerable por los problemas asociados con la llamada "globalización". La inmigración, los refugiados, el genocidio, la guerra, la convivencia interestatal y étnica, las mujeres, el mantenimiento de la identidad de pequeños grupos indígenas en organizaciones sociopolíticas más amplias, la organización o explotación de los recursos por parte de esos grupos minoritarios, la salud y el turismo, son algunos de los temas que entroncan con más claridad con las tendencias de análisis global. Se mantiene el estudio de las pequeñas comunidades, aunque es notoria la tendencia a considerar estos casos dentro de un contexto mayor, o a combinar amplios análisis cuantitativos con investigaciones de

31 Pierre Bourdieu, The Logic of Practice (Stanford, Cal.: Stanford University Press, 1990).

32 Un mundo en explosión (Barcelona: Anagrama, 1970). 
menor escala de índole cualitativa. Es injusto, en mi opinión, considerar estos ámbitos de la actividad antropológica como eclécticos, ya que bajo este calificativo desaparecen estudios de gran interés y originalidad metodológica o teórica. Tanto es así que es perfectamente posible hablar de una importante veta experimental en este tipo de trabajos aunque, quizá, puedan diferenciarse de los abiertamente experimentales por su mayor grado de empirismo o concreción de los objetivos y preguntas básicas que aspiran contestar.

Ahora bien, además de las similitudes que pueden reseñarse en dos congresos con tema semejante, celebrados con menos de un año de distancia, creo de interés señalar las diferencias que más me han llamado la atención. Muchas de ellas son atribuibles, sin duda alguna, a las organizaciones convocantes y las comunidades que engloban. No es sorprendente, por tanto, que el encuadre y consideración de las tendencias globalizadoras en los análisis se centre más en el caso de la Asociación Americana en los problemas más directamente relacionados con los Estados Unidos y su entorno, y área de mayor proyección económica y política (Latino América, el Sudeste Asiático o, de modo creciente, Europa del Este), que en la propia perspectiva que los países del "Sur" o no desarrollados puedan tener al respecto. Allí donde unos, los estadounidenses, acentúan los análisis de índole teórica, la adecuación de sus metodologías y tramas de comprensión a un espacio global y/o fragmentado, o la inclusión del tiempo; la perspectiva mundial pone un mayor énfasis en los problemas humanos de mayor transcendencia y en la búsqueda de perspectivas alternativas que incluyan el discurso minoritario más radical de las partes implicadas en las cuestiones analizadas. Por supuesto, estoy hablando de una percepción personal que no procede de un análisis estadístico y no ha de olvidarse que el predominio de las escuelas más occidentales en el quehacer antropológico es todavía fundamental.

\section{SOBRE ESPAÑA}

Hasta ahora, de modo deliberado, no he introducido de forma explícita a la antropología española en el estado de la cuestión ya que, si bien puede reclamar una larga tradición en la "etnografía" del contacto cultural y un arraigado movimiento proantropológico en el siglo xIX y primer tercio del siglo $\mathrm{xx}$, carece de una institucionalización clara hasta los años $70^{33}$.

33 Joan PRAT (coord.), "Trenta Anys de literatura antropológican, Arxiu d'Etnografia de Catalunya, 4-5 (1985-86), véanse también los artículos de Josep María Comelles sobre la historia de la antropología en España. 
Es este hecho diferencial el que ha causado que muchas de las tendencias, circunstancias y debates que se dieron en el extranjero tuvieran una repercusión muy distinta en Espana, por ello, el contexto en el que se practica y desarrolla la disciplina en España es algo distinto. No considero casual que nuestra mayor figura antropológica; Julio Caro Baroja, haya sido un intelectual al margen de cualquier institución durante la mayor parte de su carrera.

Por un lado, la relativa juventud, en el ámbito universitario y de la investigación, hace que, por ejemplo, las tendencias experimentales y las de crítica cultural, o algunas escuelas precedentes de pensamiento antropológico, estén menos presentes en nuestro entorno. Tampoco es de esperar que una preocupación por teorizar o por analizar conceptos como el de sociedad o cultura ocupen un lugar central, cuando las tareas de rescate o salvamento etnográfico, por una parte, y el desarrollo de estudios antropológicos en distintas comunidades autónomas, por otra, se convierten en los objetivos más promocionados por los gestores de la política científica. Estos últimos ámbitos pueden ser los que parecen destinados a gozar de una mayor presencia en las líneas futuras de evolución de nuestra disciplina en España. Muchas de las innovaciones teóricas y metodológicas que se incorporen a la antropología española vendrán, en mi opinión, por ese camino.

Ahora bien, la antropología española, ni se ha reducido, ni se va a limitar en el futuro a estudios basados en comunidades rurales o tradiciones populares de las distintas comunidades autónomas, ni siquiera la creciente y ferviente defensa de la etnicidad múltiple del "Estado español" agotará sus áreas prioritarias de atención; la antropología urbana, la historia de la antropología, la antropología americana (tanto del pasado como del presente), son ámbitos bien asentados ya en nuestros departamentos de antropología y ofrecen rasgos de originalidad indiscutible, por ejemplo, por el modo en el que historia y antropología han convivido juntas.

A juzgar por el debate en torno a las antropologías de España que suscitaron los artículos de Davydd Greenwood ${ }^{34}$ y Josep María Comelles y Joan Prat Carós ${ }^{35}$ en la revista Antropologia ${ }^{36}$, y el libro sobre Los españoles vistos por los antropólogos ${ }^{37}$, creo que cualquier antropólogo espanol es consciente, en la actualidad, de la dificultad y los sesgos indesea-

34 "Las antropologías de España: una propuesta de colaboración".

35 "El estado de las antropologías. Antropologías, folclores y nacionalismos en el Estado españoln.

36 Número 3, octubre de 1992.

37 Editado por María Cátedra (Madrid: Júcar, 1991). 
bles que puede introducir en el ejercicio de nuestra disciplina la incorporación acrítica de escuelas de pensamiento antropológico surgidas fuera de nuestras fronteras en contextos históricos bien diferentes. Este punto de vista no se fundamenta más que en mi propio deseo de ofrecer a la comunidad antropológica nacional e internacional una perspectiva adecuada al caso español, y significativa teóricamente para cualquier experto del mundo. Por ello creo, como muchos de los autores que expresan sus opiniones en los textos a los que me he referido, que la antropología no puede descontextualizarse de su entorno social y convertirse en una mera legitimizadora de intereses de distintos grupos de poder.

Creo que la antropología en España se encuentra en una encrucijada peculiar. Se enfrenta, por una lado, a una serie de escuelas y tendencias de pensamiento, surgidas generalmente fuera de nuestras fronteras, mientras que, por el otro, su actividad profesional está poderosamente mediatizada por un fuerte proceso de "etnogénesis", como indicaba Grenwood ${ }^{38}$, y de politización.

\section{UNA CONCLUSIÓN}

La historia se ha "antropologizado" y la antropología no teme abordar estudios de caso o trabajos de campo donde la memoria oral se combine con documentos de archivo. Los manuscritos u otros materiales escritos son hoy una realidad de la que los antropólogos hacen uso. De hecho, como mostraba en otras tradiciones, y he mencionado brevemente en el caso español, el texto es ya un campo fértil y reconocido de análisis antropológico. Ahora bien, ¿dónde queda lo social en mi panorama? ¿En qué modo es posible compatibilizar el texto, el pasado y la dimensión temporal con una disciplina formada con el objetivo de comprender la variabilidad humana?

Hoy ya no parece claro que el trabajo de campo sea la única fuente de la que nace la antropología actual, ni siquiera que la legitime frente a otras disciplinas, o que no practicarlo le haga perder su identidad primordial. Sin lugar a dudas la centralidad del trabajo de campo parece asegurada, si bien no parece ser el único ámbito de nuestra actividad durante los últimos años. Tampoco estamos sujetos a un proceso de vuelta al pasado en el que la biblioteca sustituya la realidad que existe "allí afuera"; si hemos descubierto el pasado, como he intentado mostrar, ha sido inscribiéndolo en un complejo proceso que todavía apenas alcanzamos a comprender.

38 Véase el número de Antropología, 3 (1992), arriba citado. 
Sí es claro, no obstante, que el parcial panorama de la antropología que he ofrecido en estas páginas es producto, en su diversidad, de un periodo temporal muy concreto, el que surgió de las revueltas estudiantiles y movimiento contracultural de los años 60 , más allá de nuestras fronteras, y, en España, de aquellos que, o resistieron al régimen franquista y/o lideraron la transformación democrática, y que en la actualidad son figuras indiscutibles que comienzan a ser contestadas por sus discípulos ${ }^{39}$.

Estamos ya ante una "segunda ola" surgida en circunstancias bien distintas y que se caracteriza, tal como he pretendido esbozar, de un modo diverso. No obstante las incertidumbres que generan las nuevas tendencias, es notorio resaltar una cierta recuperación del pragmatismo que lleva a integrar, en distintas dosis, el escepticismo y la crítica postmoderna dentro de un marco analítico que podría definir como de positivismo desengañado que no cree que nuestra disciplina sea capaz de construir una imagen perfecta de la realidad que pretende representar.

Los "nuevos tiempos", el nuevo paisaje intelectual que vive la antropología muestran una serie de profundos cambios. A los "lugares" tradicionales de estudio se suman hoy estudios con multitud de sitios de trabajo, preocupan las conexiones, los paralelismos y los contrastes entre grupos, culturas o espacios sin definir con claridad; tradiciones intelectuales exteriores a la antropología (como los estudios feministas, postcoloniales o los culturales) ejercen una influencia inusitada en las nuevas investigaciones ${ }^{40}$. La misma composición de los practicantes de la antropología es cada vez menos "euro-americanan. Es posible que la ruptura de las tradicionales amarras de la nave antropológica genere muchos recelos y conduzca en numerosas ocasiones a callejones sin salida, sin embargo, nunca hasta el momento había gozado esta disciplina de mejor ocasión para introducir su peculiar voz y analizar las sociedades y culturas que la vieron nacer.

\footnotetext{
39 Las etapas a las que me refiero no se corresponden con generaciones propiamente dichas. Un análisis centrado en éstas no sólo sería mucho más extenso sino, sobre todo, complejo.

40 No es, por supuesto, nueva esta situación dentro de la disciplina aunque puede argumentarse que nunca había sido más intensa la interrelación entre las influencias exteriores y los desarrollos propios dentro de la antropología.
} 
La antropología ha sufrido, desde los años 60 , una transformación dramática interpretada de modos muy diversos. Este artículo pretende ofrecer un breve perfil de la evolución de la perspectiva durante las últimas cuatro décadas, así como una visión optimista de una disciplina que, para algunos, atraviesa una crisis sin precedentes.

A personal and brief survey of the sweeping transformations that anthropology has undergone since the sixties is offered here. Its aim is to assess the discipline's new realms as well as its current strength, though this very period has been portrayed by some scholars as one of crisis.

\footnotetext{
mignets

mire ors:

250 sturs

- tomblni ist
} 